\title{
Swiss Recommendations for Systemic Therapies in Locally Advanced and Metastatic Cutaneous Squamous Cell Carcinoma
}

\author{
Mirjam Nägeli*, Joanna Mangana ${ }^{1 *}$, Roger von Moos², Lukas Flatz³, Robert E. Hunger ${ }^{4}$, Rastine Merat ${ }^{5}$, Olivier Gaide ${ }^{6}$, \\ Marco Siano ${ }^{7}$, Reinhard Dummer ${ }^{1 *}$
}

ABSTRACT

Cutaneous squamous cell carcinoma (cSCC) is the second most frequent type of non-melanoma skin cancer. Its incidence has been rising in recent years, with the highest rate reported in Switzerland compared to other countries in Europe. While the majority of cSCC cases are low-grade tumors with an excellent prognosis following surgical excision, a minority of cSCC lesions (approximately $5 \%$ of patients) progress to locally advanced cSCC (lacSCC) or distant metastatic disease (mcSCC), both of which have an unfavorable prognosis. Recent evidence from studies with checkpoint immunotherapy has changed the systemic treatment landscape for lacSCC and mcSCC patients. While both programmed death-1 (PD-1) receptor inhibitors cemiplimab and pembrolizumab are approved by the FDA, cemiplimab is the only approved systemic therapy for the treatment of nonresectable advanced cSCC in the EU and was recently also approved in Switzerland. Based on the latest evidence from randomized clinical trials, national consensus recommendations for the systemic treatment of advanced cSCC have been defined. For classification and optimal management of patients with lacSCC or mcSCC, an interdisciplinary tumor board discussion should be mandatory. Difficult-to-treat advanced cSCC patients should be referred to and treated by specialized centers. These Swiss recommendations provide guidance for the management of patients aged $\geq 18$ years with lacSCC or mcSCC, specifically systemic therapy with a PD-1 inhibitor in the first-line setting. These up-to-date recommendations will also help Swiss physicians in their decision-making and address treatment variability in Swiss clinical practice.

Keywords: cSCC, locally advanced, metastatic, systemic treatment, immunotherapy, cemiplimab

\section{INTRODUCTION}

The incidence of cutaneous squamous cell carcinoma (cSCC) is rising due to the increased longevity of at-risk patients. ${ }^{1,2}$ To date, approximately $20 \%$ of all non-melanoma skin cancers (NMSCs) that arise each year can be attributed to cSCC, and 2-5\% of these will ultimately metastasize. ${ }^{3}$ Switzerland has one of the highest incidences of NMSC in Europe, ${ }^{4}$ with an estimated 25,000 patients per year affected. ${ }^{5}$ The rising incidence together with the nonnegligible patient and economic burden and mortality rate highlights the importance and the clinical relevance of treatments for advanced cSCC. ${ }^{6}$

In Switzerland, there is currently no consensus first-line systemic treatment for patients who have locally advanced cSCC (lacSCC) or distant metastatic disease (mcSCC). ${ }^{7-9}$ There are several major classes of systemic agents used to treat patients, including immunotherapy, anti-epidermal growth factor receptor (EGFR) therapy, and chemotherapy. ${ }^{3}$ Recent studies with immune checkpoint inhibitors, also called programmed cell death protein 1 (PD-1) inhibitors, have yielded promising outcomes in advanced cSCC with approximately $50 \%$ objective response rates (ORR), and have led to the FDA, EMA and Swissmedic approval of the anti-PD-1 monoclonal antibody cemiplimab for unresectable high-risk cSCC. ${ }^{10-12} \mathrm{~A}$ systematic review of clinical studies with regard to efficacy, side effects and sustainability of therapeutic methods used in Swiss practice is therefore warranted.

The aim of these Swiss recommendations is to provide Swiss physicians with accepted, evidence-based decision support for the selection and implementation of systemic therapy
${ }^{1}$ Department of Dermatology, University Hospital Zurich, Zurich, Switzerland ${ }^{2}$ Department of Oncology and Haematology, Graubünden Cantonal Hospital, Chur, Switzerland ${ }^{3}$ Clinic of Dermatology, Venereology and Allergology, St.Gallen Hospital, St. Gallen, Switzerland

4Department of Dermatology, Inselspital, Bern University Hospital, Bern, Switzerland

${ }^{5}$ Division of Dermatology and Venereology, Geneva University Hospital, Geneva, Switzerland

${ }^{6}$ Department of Radiation Oncology, Lausanne University Hospital, Lausanne, Switzerland

7 Interdisciplinary Cancer Service, HRC Hospital Riviera-Chablais, Rennaz, Switzerland

Corresponding Author(s):

Dr Mirijam Nägeli

Department of Dermatology, University Hospital Zurich, Zurich, Switzerland Email: mirjam.naegeli@usz.ch Tel: +41442551111 Dr Joanna Mangana Department of Dermatology, University Hospital Zurich, Zurich, Switzerland Email: joanna.mangana@usz.ch Tel: +41 442551111

DOI: 10.36000/hbT.OH.2021.08.031. ISSIN: 2673-2092 (Print) and 2673-2106 (Online)

This article was received on May 25, 2021

This article was accepted on May 30, 2021

This article was published on June 11, 2021

Nägeli M et al. Swiss Recommendations for Systemic Therapies in Locally Advanced and Metastatic Cutaneous Squamous Cell Carcinoma. healthbook TIMES Onco Hema. 2021; (8): 12-21 
in patients with locally advanced or distant metastatic cSCC. These recommendations may also improve standards-of-care in cSCC when patients are treated or managed in clinical practices outside Swiss centers of excellence. Study results of the recommended systemic therapies with regard to benefits and risks are also described to further support physicians in the decision-making process.

\section{LEVEL OF EVIDENCE}

The level of evidence for included studies is graded according to the Oxford classification (Oxford Centre for EvidenceBased Medicine 2011 levels of evidence). ${ }^{13}$

- Level of evidence I: Meta-analysis, phase I and phase II cohort studies

- Level of evidence II: Guideline adaptation, systematic review and meta-analysis, retrospective study

- Level of evidence III: Review, prospective study, retrospective study, guidelines

- Level of evidence IV: Case-series, case-controlled studies, or historically controlled studies

The grades of recommendations are classified as follows:

- A: Strong recommendation (shall)

- B: Recommendation (should)

- C: Weak recommendation (may/can)

- X: Should not be recommended

- 0: Recommendation pending: Not available currently or not enough evidence to give a recommendation in favor or against.

\section{DEFINITION OF CSCC}

Herein, lacSCC shall be defined as non-metastatic cSCC, not amenable to either surgery or radiotherapy with reasonable hope for cure, because of multiple recurrences, large extension, bone erosion or invasion, or deep infiltration beyond subcutaneous tissue into muscle or along nerves, or else tumors in which curative resection would result in unacceptable complications, morbidity or deformity. ${ }^{14}$ Metastatic cSCC (mcSCC) includes loco-regional metastatic cSCC with intransit metastases or metastasis to regional lymph nodes, or distant metastatic cSCC requiring systemic treatments. ${ }^{14}$ Table 1 shows the staging classification used for both lacSCC and mcSCC based on the American Joint Committee on Cancer (AJCC)/International Union Against Cancer (UICC) tumor-node-metastasis (TNM) classification. This staging system is not without limitations, and Swiss physicians should only use this tool as part of an interdisciplinary approach to help classify patients into either low- or high-risk cSCC categories.

\section{AN INTERDISCIPLINARY APPROACH IS NEEDED FOR TREATING CSCC PATIENTS}

Every treating physician should start with classification of the cSCC to be treated, i.e., does the patient have a high- or low-risk tumor. Classification should also include
Table 1. Staging based on AJCC/UICC TNM classification, 2017.

$\mathrm{T} 1$, primary tumor $\leq 2 \mathrm{~cm}$ in greatest dimension; $\mathrm{T} 2$, primary tumor $>2 \mathrm{~cm}$ and $<4 \mathrm{~cm}$ in greatest dimension; $T 3$, primary tumor $>4 \mathrm{~cm}$ in greatest dimension or minor bone erosion or perineural invasion (PNI) or deep invasion (defined as invasion beyond the subcutaneous fat or $>6 \mathrm{~mm}$ ); 14 , primary tumor with gross cortica bone/marrow invasion or with axial skeleton invasion including foraminal involvement and vertebral foramen involvement to the epidural space; NO, no regional lymph node metastasis; N1, metastasis in a single ipsilateral lymph node $\leq 3 \mathrm{~cm}$ in greatest dimension without extranodal extension (ENE); N2, metastasis in single, ipsilateral lymph node $\leq 3 \mathrm{~cm}$ with ENE or, $>3 \mathrm{~cm}$ in greatest dimension without ENE; N3, metastasis dimension with ENE or multiple ipsilateral or with contralateral or bilateral node(s) with ENE; MO, no distant metastasis: M1 distant metastasis: AJCC, UICC TNM, American Joint Committee on Cancer (AJCOV International Union Against Cancer (UICC) tumor-node-metastasis. Adapted from Stratigos et al. 2020.13

\begin{tabular}{|l|c|c|c|}
\hline Stage 0 & Tis & N0 & M0 \\
\hline Stage I & T1 & NO & MO \\
\hline Stage II & T2 & NO & MO \\
\hline Stage III & T3 & NO & MO \\
\hline Stage IVA & T1, T2, T3 & N2, N3 & MO \\
& T4 & Any N & \\
\hline Stage IVB & Any T & Any N & M1 \\
\hline
\end{tabular}

whether the tumor is mcSCC or lacSCC. It is important to have experienced surgeons and radio-oncologists as part of an interdisciplinary tumor board to discuss and classify individual patient cases. All decisions for systemic therapy should be made by an interdisciplinary tumor board. Difficultto-treat patients with lacSCC or mcSCC should be referred to and treated by specialized centers. Furthermore, and in addition to its definition and classification, it is important to note that tumor kinetics are often more rapid for lacSCC. ${ }^{7}$ For example, lacSCC often emerges from relapsing tumors, e.g. it can emerge as a fast, advancing tumor and/or as a rapid relapse (relapse within 3 months) of a previous successfully resected tumor. Various risk factors for high-risk tumors should also be considered by Swiss physicians, to help determine which atrisk patients should be considered for early systemic treatment (Table 2). The treatment management pathway for cSCC in Switzerland is shown in Figure 1.

Table 2. Prognostic risk factors for developing/recurrence of advanced cSCC. Adapted from Seidl-Philipp et al. $2020^{56}$ and Stratigos et al. 202057.

\begin{tabular}{|l|}
\hline High-risk factors \\
Clinical factors \\
\hline - Tumor size (diameter) $>2 \mathrm{~cm}$ \\
- Tumor site on ear, lip or areas of long-lasting chronic ulcers or \\
inflammation \\
- Recurrent lesions \\
- Incomplete excision \\
- Host immunosuppression \\
\hline Pathological factors \\
\hline - Depth of invasion $\geq 6$ mm if host immunocompetent, \\
- Acantholytic, spindle cell or desmoplastic histological subtype \\
- Moderately or poorly differentiated tumors \\
- Perineural involvement
\end{tabular}




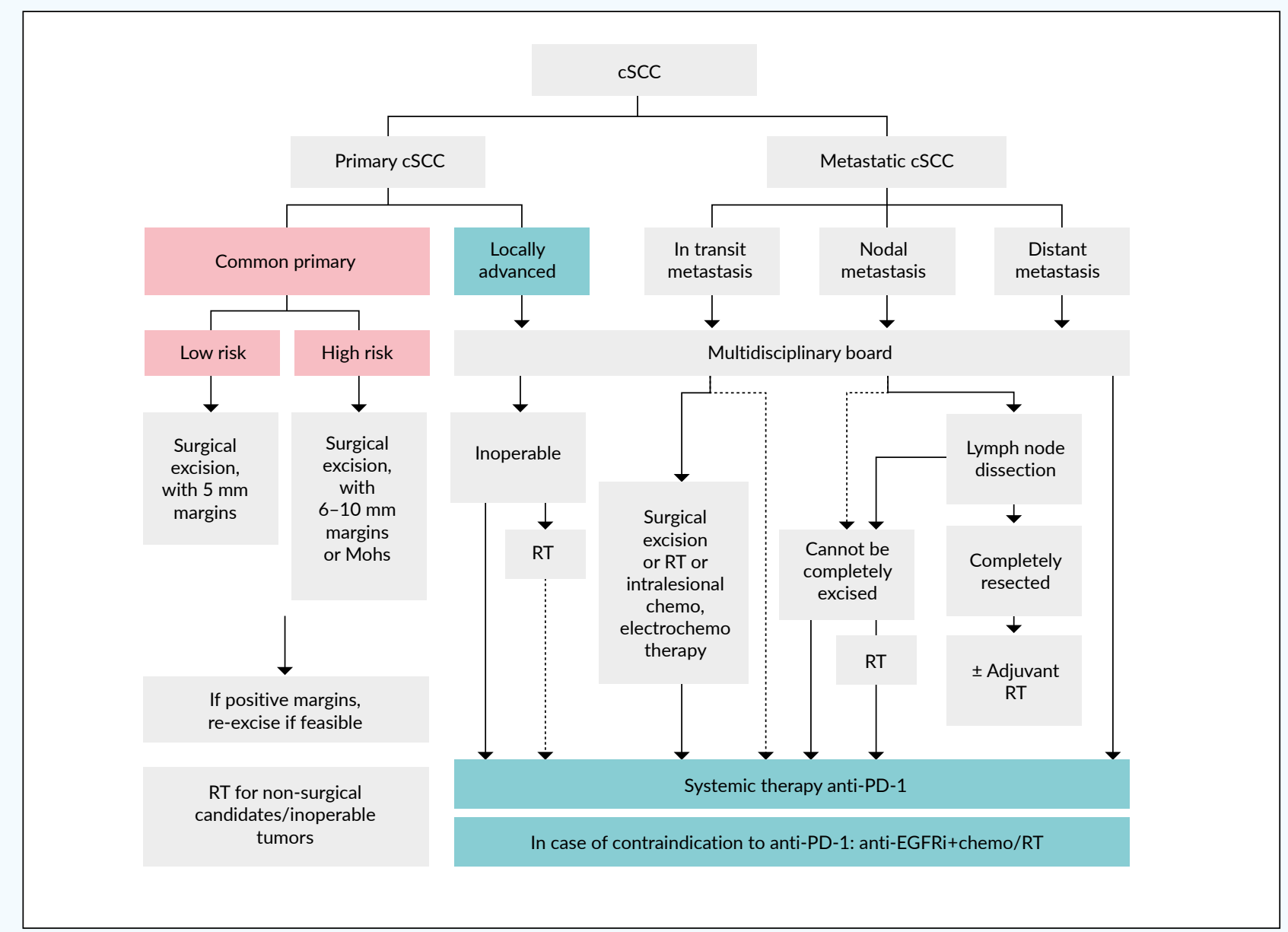

Figure 1. Swiss treatment algorithm for cSCC. Adapted from Stratigos et al. 2020.

\section{TREATMENT OF COMMON PRIMARY CSCC}

Briefly, the first-line approach for primary cSCC involves surgical excision of the tumor with careful assessment of skin margins regardless of the age group and anatomic location (Table 3). ${ }^{7}$ Postoperative assessment of the resection margins is required as standard-of-care during conventional surgery, with recommended $5 \mathrm{~mm}$ or 6-10 $\mathrm{mm}$ safety margins for low- or high-risk lesions, respectively. ${ }^{7}$ Conventional surgery is a preferred approach for low-risk cSCC tumors. ${ }^{7}$ En bloc excision with subsequent skin grafting is a suitable option for patients with a cluster of multiple cSCCs (e.g. on the scalp). ${ }^{7}$ Re-excision of positive margins should be performed for all operable cases (Table 3). ${ }^{7}$

There are two main types of surgical procedure, conventional surgery and micrographically controlled surgery (MCS), both are indicated regardless of patient age and anatomic location of the tumor. ${ }^{7}$

MCS is a particularly effective treatment for high-risk cSCC. ${ }^{7}$ It involves removing serial horizontal sections of the tumor margin in order to spare as much tissue margin as possible while minimizing the risk of recurrence. ${ }^{7}$ Two techniques of
MCS are available in Switzerland: MMS (Mohs Micrographic Surgery) which uses frozen sections, and 3D histology or "slow Mohs" which uses paraffin sections. ${ }^{7}$ Surgical removal by MCS is more time-consuming, labor-intensive and therefore more expensive, but achieves higher rates $(>90 \%)$ of R0 resection (microscopic disease-free margins) and lower rates ( $\leq 4 \%$ vs. $3.1-8.0 \%$ ) of recurrence, compared to conventional surgery. ${ }^{7}$ For these reasons, MMS may be preferred to conventional surgery for excision of certain cSCCs, e.g. those on the head and neck with a high recurrence rate. ${ }^{7}$ Another major advantage of MMS is same-day tumor removal and reconstructive surgery.

Regular physical examination, including inspection of the entire skin and inspection and palpation of the excision site, the in-transit route and the regional lymph nodes, should be part of the follow-up for all patients. ${ }^{7}$ For patients with a low risk of recurrence or new skin cancers, it is recommended that they have a clinical examination every 6-12 months for 5 years. ${ }^{7}$ For high-risk primary cSCC, e.g. for patients with a risk of local recurrence or new skin cancers and risk of regional metastases, follow-up every 3-6 months for 2 years or every 6-12 months for 3 to 5 years, respectively, is recommended, and annually thereafter. 
Table 3. Summary of Swiss recommendations for surgical and non-surgical primary cSCC treatments.

cSCC, cutaneous squamous cell carcinoma; MMS, Mohs micrographic surgery. Adapted from Stratigos et al. 2020.7

\begin{tabular}{|c|c|c|}
\hline Recommendation & $\begin{array}{l}\text { Grade of } \\
\text { recommendation }\end{array}$ & $\begin{array}{l}\text { Level of } \\
\text { evidence }\end{array}$ \\
\hline \multicolumn{3}{|l|}{ Surgical procedures: } \\
\hline $\begin{array}{l}\text { R1. Standard cSCC surgical removal with complete excision (RO) and histological confirmation of } \\
\text { peripheral and deep excision margins }\end{array}$ & A & 2 \\
\hline R2. In case of positive margins, a re-excision should be performed, for operable cases & A & 2 \\
\hline $\begin{array}{l}\text { R3. Low-risk and high-risk cSCC should be excised with a clinical safety margin of } 5 \mathrm{~mm} \text { and } 6-10 \mathrm{~mm} \\
\text { or by MMS, respectively }\end{array}$ & B & $2-3$ \\
\hline $\begin{array}{l}\text { R4. For regional lymph nodes, dissection should be performed in clinically or radiologically detected } \\
\text { lymph node metastasis that is confirmed with cytology or biopsy; the extent of surgical resection } \\
\text { should be determined by the surgeon in collaboration with the interdisciplinary tumor board e.g., } \\
\text { lymph node region }\end{array}$ & B & 3 \\
\hline R5. Elective lymph node dissection should not be performed for cSCC & $x$ & 4 \\
\hline \multicolumn{3}{|l|}{ Radiotherapy: } \\
\hline $\begin{array}{l}\text { R6. Primary radiotherapy should be considered as an alternative to surgery for inoperable or } \\
\text { difficult-to-operate tumors or in the absence of consent to surgical excision }\end{array}$ & B & 3 \\
\hline $\begin{array}{l}\text { R7. Adjuvant radiotherapy should be considered for patients with head and neck regional nodal } \\
\text { metastases and extracapsular extension }\end{array}$ & B & 3 \\
\hline $\begin{array}{l}\text { R8. Postoperative radiotherapy should be considered after surgical excision for cSCC with positive } \\
\text { margins and for which re-excision is not possible }\end{array}$ & B & 3 \\
\hline
\end{tabular}

SURGICAL TREATMENT FOR REGIONAL NODAL DISEASE Following clinically or radiologically detected lymph node-positive cSCC, i.e. confirmed with cytology or biopsy, radical lymph node dissection of all affected areas should be performed (Table 3). ${ }^{7}$ The interdisciplinary tumor board should decide the extent of dissection for each patient case, as well as nonoperative therapies when surgery is contraindicated or if the patient refuses surgical treatment. ${ }^{7}$ Due to the rarity of nodal metastases, elective lymph node dissection is not recommended for lymph node-negative cSCC. ${ }^{7}$ For patients with high risk of regional and distant metastases, follow-up every 3 months for 5 years and every 6-12 months thereafter is recommended.

\section{NON-SURGICAL TREATMENTS FOR SELECTED PRIMARY CSCC CASES}

In Switzerland, alternative destructive modalities such as curettage and electrodessication, photodynamic therapy (PDT), cryotherapy and lasers are not recommended for primary invasive cSCC. ${ }^{7}$ For non-invasive, i.e. in situ, cSCC, these approaches could be used.

\section{ADJUNCT RADIOTHERAPY}

Radiotherapy is a considerable alternative for patients with primary cSCC for whom curative surgery is not possible (e.g. due to comorbidities, or if the patient declines surgery) or when surgery could cause disfigurement or a poor functional outcome (Table 3). ${ }^{7}$ For small cSCCs (e.g., diameter $<1 \mathrm{~cm}$ ), definitive primary radiotherapy is a suitable alternative to surgery. ${ }^{7}$ Long-term effects are very good in some areas, e.g. the periocular region, but less so for areas such as the ear. For regional nodal metastases and extracapsular extension of head and neck cSCCs, adjuvant radiotherapy should be considered (Table 3). ${ }^{7}$ Postoperative radiotherapy is also an option for cSCC with positive margins after surgical excision if reexcision is not possible.

\section{SYSTEMIC TREATMENTS FOR ADVANCED CSCC}

It is important to determine which cSCC patients should receive systemic therapy after interdisciplinary tumor board discussion. For example, patients with advanced cSCC, including regional node involvement or metastases to distant tissues or organs, may not respond to surgery or radiation, so earlier systemic therapy is needed. ${ }^{7}$ Systemic treatment options include immunotherapy, epidermal growth factor receptor (EGFR) inhibitors, chemotherapy (platinum-based chemotherapy was used as the sstandard of care in the past), and electrochemotherapy. ${ }^{7}$ Notably, Swiss physicians should offer advanced cSCC patients to be treated in a clinical trial, whenever possible.

\section{IMMUNOTHERAPY WITH IMMUNE CHECKPOINT INHIBITORS}

Clinical trials with immune checkpoint inhibitors (ICIs) were designed based on the rationale that expression of cell surface programmed cell death 1 receptor/ligand (PD-1/PD-L1) was linked to poor clinical outcomes in cSCC..$^{15,16}$ Until the recent introduction of ICIs, there was no approved agent for lacSCC 
and mcSCC, and available treatments had very limited efficacy with significant adverse reactions. ${ }^{7}$ The monoclonal PD-1 inhibitor cemiplimab is to date the only approved systemic therapy in cSCC in Switzerland; it is currently indicated for patients with lacSCC or mcSCC who are not candidates for curative surgery or curative radiation (Table 4). ${ }^{7,10}$ It is important to consider comorbidities for each patient as part of the interdisciplinary team discussions, since some patients, e.g. immunocompromised or post-organ transplantation or patients with autoimmune diseases, were excluded from clinical trials and ICI treatment. Since solid organ transplant recipients (sOTRs) have the highest incidence of SCC, two studies with anti-PD-1 have been approved for renal transplant patients with cancer in the U.S. as these patients are eligible for hemodialysis in case of organ rejection. There are more and more case reports about sOTR and anti-PD-1 therapy, and each individual case should be discussed with the interdisciplinary team in case of a high risk of organ rejection with immunotherapy. ${ }^{17-20}$ Moreover, there are no convincing reasons to date why patients with hematological diseases such as monoclonal gammopathy of undetermined significance (MGUS) or chronic lymphatic leukemia (CLL) should not be treated with PD-1 inhibitors. Pembrolizumab, another PD-1 inhibitor, is currently being investigated in cSCC clinical studies, and was recently approved for cSCC by the FDA. ${ }^{7}$ Except for cemiplimab, all other systemic treatments are currently used off-label in Europe as well as in Switzerland. ${ }^{7}$

\section{EGFR INHIBITORS}

The epidermal growth factor receptor (EGFR) plays a key role in the activation of multiple downstream signalling pathways involved in cell proliferation, apoptosis, invasion, angiogenesis and metastasis. ${ }^{21}$ In squamous cells, EGFR plays an important role in regulating the $\mathrm{RAS} / \mathrm{MAPK}, \mathrm{PI} 3 \mathrm{~K} / \mathrm{AKT}$ and phospholipase $\mathrm{C}$ pathways, and is strongly associated with the development of cSCC. ${ }^{22}$ EGFR overexpression in cSCC has been reported as $43 \%,{ }^{23}$ and appears also to have prognostic implications associated with lymph node metastasis and progression proportional to the metastatic risk. ${ }^{7,23-25}$ However, a low frequency of somatic mutations of EGFR (2.5-5\%) in cSCC has been found, i.e. anti-EGFR therapy may be suitable for a small subset of cSCC patients with genetic activation of EGFR by mutation or amplification..$^{26,27}$

The EGFR is highly expressed in many epithelial tumors, including cSCC of the head and neck. ${ }^{28}$ Two monoclonal EGFR-targeting antibodies, cetuximab and panitumumab, have been evaluated in patients with cSCC. ${ }^{29,30}$ For patients with lacSCC and mcSCC who have failed to respond or are intolerant to immunotherapy, cetuximab may be used as second-line treatment after cemiplimab (first-line), preferably in combination with chemotherapy or radiotherapy (Table 4). ${ }^{7}$ Efficacy with EGFR inhibitors combined with chemotherapy has been shown in advanced cSCC but data is available mainly from clinical case series, i.e. there are no prospective randomized trials that can provide details about durability of responses. Cetuximab may be preferred as the second-line agent for elderly patients with comorbidities, who may not tolerate chemotherapy. ${ }^{7}$ Other available targeted EGFR inhibitors include small-molecule receptor tyrosine kinase inhibitors such as erlotinib, gefitinib, and lapatinib.

\section{CHEMOTHERAPY AND ELECTROCHEMOTHERAPY}

Although there are no systemic chemotherapies approved for treating advanced cSCC patients, platinum-based agents may be used in the second-line setting when patients fail to respond or are intolerant to anti-PD-1 immunotherapy. ${ }^{7}$ Chemotherapy in combination with EGFR inhibitors or radiotherapy may be more effective. ${ }^{7}$ Although electrochemotherapy (ECT) is often available in most centers, there are no prospective, randomized studies about its long-term effectiveness in advanced cSCC. Electrochemotherapy may be considered by the interdisciplinary tumor board and reserved for a very select number of patients, treated at specialized Swiss centers (Table 4).

Table 4. Summary of Swiss systemic therapy recommendations for advanced cSCC.

mcSCC, metastatic cutaneous squamous cell carcinoma; lacSCC, local advanced cutaneous squamous cell carcinoma; PD-1, programmed death receptor 1; EGFR, epidermal growth factor receptor. Adapted from Stratigos et al. 2020.7

\begin{tabular}{|c|c|c|}
\hline Swiss Recommendation & $\begin{array}{l}\text { Grade of } \\
\text { recommendation }\end{array}$ & $\begin{array}{l}\text { Level of } \\
\text { evidence }\end{array}$ \\
\hline \multicolumn{3}{|l|}{ Surgical procedures: } \\
\hline $\begin{array}{l}\text { R1. Patients with mcSCC or lacSCC who are not candidates for curative surgery or curative radiation } \\
\text { should receive first-line treatment with an anti-PD-1 antibody (Note: Cemiplimab is currently the only } \\
\text { approved medication in Europe, while pembrolizumab is investigated in clinical studies) }\end{array}$ & $A$ & 2 \\
\hline $\begin{array}{l}\text { R2. The anti-EGFR cetuximab may be used in the second-line setting for patients with lacSCC and } \\
\text { mcSCC who have failed to respond or are intolerant to immunotherapy; Cetuximab combined with } \\
\text { chemotherapy or radiotherapy is favored over cetuximab monotherapy }\end{array}$ & C & 3 \\
\hline $\begin{array}{l}\text { R3. Chemotherapy may be used in the second-line setting when patients fail to respond or are } \\
\text { intolerant to anti-PD-1 immunotherapy; Platinum-based chemotherapy is preferred, and chemotherapy } \\
\text { may be more effective when used in combination with EGFR inhibitors or radiotherapy }\end{array}$ & C & $3-4$ \\
\hline
\end{tabular}




\section{SWISS RECOMMENDATIONS FOR USE OF SYSTEMIC THERAPIES}

\section{PD-1 INHIBITORS}

Checkpoint antibody inhibitors such as anti-PD-1 agents function as tumor suppressing factors via modulation of immune cell to tumor cell interaction. ${ }^{31}$ Cemiplimab, an intravenous human monoclonal antibody directed against PD-1, blocks T-cell inactivation and enhances the immune system's anti-tumor response. The FDA approved cemiplimab in $2018^{11}$ closely followed by the EMA in $2019^{10}$ for treating lacSCC or distant metastatic disease in which curative surgery or radiotherapy is not feasible. Swissmedic approved cemiplimab in May 2020. Initial approval was based on the results of two clinical trials (NCT02383212 and NCT02760498; Table 5). ${ }^{32,33}$ Migden et al. (2018) reported integrated data from an open-label, multicenter phase I cemiplimab study that included an expansion cohort of 26
lacSCC and mcSCC patients, as well as a nonrandomized global phase II study of $59 \mathrm{mcSCC}$ patients. ${ }^{32}$ In phase I and phase II, cemiplimab was administered intravenously at $3 \mathrm{mg} / \mathrm{kg}$ every 2 weeks for up to 48 weeks (phase I) or 96 weeks (phase II). ${ }^{32}$ The objective response rate (ORR) was 50\% (95\% CI: 30-70) and 47\% (95\% CI: 34-61) for phase I and phase II cohorts, respectively. ${ }^{32}$ Among responders with a median follow-up of 7.9 months, $61 \%$ of mcSCC patients in the phase II cohort had durable disease control with an acceptable safety profile, meaning that they avoided progressive disease for at least 105 days. ${ }^{32}$ The results of the pivotal single-arm phase II cemiplimab study, also reported by Migden et al. (2020), enrolled a total of 78 lacSCC patients without nodal or distant metastasis from 25 outpatient clinics across Australia, Germany, and the U.S. between June 14, 2016, and April 25, 2018. ${ }^{33}$ Patients received cemiplimab $3 \mathrm{mg} / \mathrm{kg}$ every 2 weeks for up to 96 weeks. The ORR (primary endpoint) was observed in 34 of 78 patients (44\%; $95 \% \mathrm{CI}$;

Table 5. Response outcomes of prospective trials of systemic therapies for the treatment of advanced cSCC in Switzerland. Disclaimer: This table is an illustration and not a direct comparison of the studies.

CSCC, cutaneous squamous cell carcinoma; lacSCC, locally advanced cSCC; mcSCC, metastasis CSCC; CR, complete response; i.v., intravenous; PR, partial response; ORR, objective response rate (CR + PR); PFS, progression free-survival; OS, overall survival; r/mcSCC, recurrent-metastatic cSCC; NCT ID, national clinical trial identification. Adapted from Stratigos et al. 2020.,12

\begin{tabular}{|c|c|c|c|c|c|c|c|c|}
\hline $\begin{array}{l}\text { Clinical } \\
\text { study }\end{array}$ & Trial design & $\begin{array}{l}\text { Patients } \\
\text { (N) }\end{array}$ & $\begin{array}{l}\text { Type of } \\
\text { cSCC }\end{array}$ & $\begin{array}{l}\text { Treatment } \\
\text { scheme }\end{array}$ & Response & Survival & NCT ID & Ref. \\
\hline \multicolumn{9}{|c|}{ Immune checkpoint inhibitors - level of evidence 2} \\
\hline $\begin{array}{l}\text { Migden et al. } \\
2018\end{array}$ & $\begin{array}{l}\text { Phase I, } \\
\text { open-label, } \\
\text { multicenter }\end{array}$ & 26 & $\begin{array}{l}10 \operatorname{lacSCC} \\
16 \operatorname{mcSCC}\end{array}$ & $\begin{array}{l}\text { Cemiplimab: } \\
3 \text { mg/kg i.v. } \\
\text { over } 30 \text { min } \\
\text { every } 2 \text { weeks } \\
\text { for up to } 48 \\
\text { weeks }\end{array}$ & $\begin{array}{l}\text { Best ORR: } \\
50 \% \text {; } \\
13 \text { patients } \\
\text { with PR }\end{array}$ & Not stated & NCT02383212 & 32 \\
\hline \multirow{3}{*}{$\begin{array}{l}\text { Rischin et al. } \\
2021 \\
\text { (EMPOWER) }\end{array}$} & \multirow{3}{*}{$\begin{array}{l}\text { Phase II, } \\
\text { open-label, } \\
\text { non- } \\
\text { randomized, } \\
\text { multicenter, } \\
\text { pivotal study }\end{array}$} & \multirow{3}{*}{193} & $59 \mathrm{mcSCC}$ & $\begin{array}{l}\text { Cemiplimab: } \\
3 \text { mg/kg i.v. } \\
\text { over } 30 \text { min } \\
\text { every } 2 \text { weeks } \\
\text { for up to } 96 \\
\text { weeks }\end{array}$ & $\begin{array}{l}\text { Best ORR: } \\
51 \% \text {; } \\
20 \% \text { CR, } \\
31 \% \text { PR }\end{array}$ & \multirow{3}{*}{$\begin{array}{l}\text { Median OS for all } 3 \text { groups: } \\
\text { not yet reached } \\
\text { Estimated probability of } \\
2 \text {-year OS for all } 3 \text { groups: } \\
73 \% \\
\text { Median PFS for all } 3 \text { groups: } \\
18.5 \text { months }\end{array}$} & \multirow{3}{*}{ NCT02760498 } & \multirow{3}{*}{58} \\
\hline & & & 78 lacSCC & $\begin{array}{l}\text { Cemiplimab: } \\
3 \text { mg/kg i.v. } \\
\text { over } 30 \text { min } \\
\text { every } 2 \text { weeks } \\
\text { for up to } 96 \\
\text { weeks }\end{array}$ & $\begin{array}{l}\text { Best ORR: } \\
45 \% \text {; } \\
\text { 13\% CR, } \\
32 \% \text { PR }\end{array}$ & & & \\
\hline & & & $56 \mathrm{mcSCC}$ & $\begin{array}{l}\text { Cemiplimab: } \\
\text { fixed dose } \\
\text { ( } 300 \mathrm{mg}) \text { every } \\
3 \text { weeks for up } \\
\text { to } 54 \text { weeks }\end{array}$ & $\begin{array}{l}\text { Best ORR: } \\
46 \% \\
20 \% \text { CR: } \\
27 \% \text { PR }\end{array}$ & & & \\
\hline \multirow{4}{*}{$\begin{array}{l}\text { Grob et al. } \\
2021 \text { (KEY- } \\
\text { NOTE-629) }\end{array}$} & \multirow{4}{*}{$\begin{array}{l}\text { Phase II, } \\
\text { single-arm, } \\
\text { open-label, } \\
\text { non- } \\
\text { randomized } \\
\text { study }\end{array}$} & \multirow{4}{*}{159} & \multirow{2}{*}{54 lacSCC } & \multirow{2}{*}{$\begin{array}{l}\text { Pembrolizumab } \\
\text { (200 mg i.v. } \\
\text { every } 3 \text { weeks) } \\
\text { for up to } 35 \\
\text { cycles }\end{array}$} & \multirow{2}{*}{$\begin{array}{l}\text { Best ORR: } \\
35 \% \text {; } \\
11 \% \mathrm{CR} \\
25 \% \mathrm{PR}\end{array}$} & $\begin{array}{l}\text { Median OS: } 23.8 \text { months; } \\
\text { OS rate at } 12 \text { months: 61\% }\end{array}$ & \multirow{4}{*}{ NCT03284424 } & \multirow{4}{*}{58} \\
\hline & & & & & & $\begin{array}{l}\text { Median PFS: } 5.7 \text { months; } \\
\text { PFS rate at } 12 \text { months: } 36 \%\end{array}$ & & \\
\hline & & & \multirow{2}{*}{$105 \mathrm{r} / \mathrm{mcSCC}$} & \multirow{2}{*}{$\begin{array}{l}\text { Pembrolizumab } \\
\text { ( } 200 \text { mg i.v. } \\
\text { every } 3 \text { weeks) } \\
\text { for up to } 35 \\
\text { cycles }\end{array}$} & \multirow{2}{*}{$\begin{array}{l}\text { Best ORR: } \\
50 \% \text {; } \\
17 \% \mathrm{CR} \\
33 \% \mathrm{PR}\end{array}$} & $\begin{array}{l}\text { Median OS: not yet reached; } \\
\text { OS rate at } 12 \text { months: } 74 \%\end{array}$ & & \\
\hline & & & & & & $\begin{array}{l}\text { Median PFS: not yet reached; } \\
\text { PFS rate at } 12 \text { months: } 54 \%\end{array}$ & & \\
\hline
\end{tabular}


32-55), with complete response (CR) seen in 10 patients $(13 \%) .{ }^{33}$ Stable disease (SD) was observed in an additional 28 patients (36\%). ${ }^{33}$ Median duration of follow-up was 9.3 months and median duration of response had not been reached at data cut-off (October 10, 2018). Estimated 12-month progression-free survival was $58 \%$ and estimated 12 -month overall survival was $93 \% .{ }^{33}$ Cemiplimab showed an acceptable safety profile, with Grade 3-4 treatment-emergent adverse events occurring in $44 \%$ of patients and serious treatmentemergent adverse events occurring in $29 \%$ of patients. ${ }^{33}$ The importance of anti-PD-1 therapy has been further supported by data on treatment with pembrolizumab. The multicenter, open-label, non-randomized phase II EMPOWER-cSCC-1 trial included 193 advanced cSCC patients with a median age of 72 years. At the American Society of Clinical Oncology 2020 (ASCO20) Virtual Congress, the $\sim 1$-year follow-up from this large prospective study in advanced cSCC was presented. The results showed that patients treated with cemiplimab demonstrated an objective response rate (ORR) of $46.1 \%$. Among patients who had received prior systemic anti-cancer therapy, ORR was $41.5 \%$ and $48.4 \%$ in those who had not. ${ }^{34}$ Results from the post-hoc analysis of phase II trial presented at the ASCO20 Virtual Congress further showed that improvement in global health status/health-related quality of life (HRQL) was observed as early as cycle 3 with clinically meaningful benefit through cycle 12 in advanced cSCC patients treated with cemiplimab. ${ }^{35}$

Results of the phase II CARSKIN study with pembrolizumab on 39 patients with unresectable cSCC, with no prior systemic treatment and a median age of 80 years, showed a response rate of $38.5 \%$ and a median progression-free survival of 8.4 months (NCT02883556). ${ }^{36}$ Additionally, in KEYNOTE-629 (NCT03284424), the efficacy and safety of pembrolizumab is being evaluated in adults with recurrent/ mcSCC or lacSCC. ${ }^{12,37}$

Use of anti-PD-1 agents in the adjuvant setting are not covered in these Swiss recommendations since clinical trials are ongoing. ${ }^{38-40}$

Although there are no evidence-based data on when to cease the treatment with the anti-PD-1 antibodies, we suggest similar application as for melanoma.

For metabolic CR (mCR)/CR, patients should be treated for 6 months after CR has been achieved, and for metabolic partial response $(\mathrm{mPR}) / \mathrm{PR}$, patients should be treated for 2 years.

\section{EGFR INHIBITORS}

Cetuximab is a humanised monoclonal antibody directed against the extracellular domain of EGFR, and is approved in Europe for the treatment of patients with lacSCC of the head and neck in combination with radiation therapy and patients with recurrent/mcSCC in combination with platinum-based chemotherapy. ${ }^{22,41}$ Off-label use has included cetuximab monotherapy ${ }^{42-44}$ or cetuximab combined with radiotherapy or cisplatin, ${ }^{43,45-49}$ for advanced cSCC in a small number of patients in prospective studies or patient cases. Hence, there is a paucity of data with EGFR inhibitors in Europe as well as in Swiss clinical practice. In advanced cSCC (lacSCC and mcSCC patients), first-line cetuximab monotherapy demonstrated a disease control rate (DCR) of $69 \%$ at 6 weeks (Table 4). ${ }^{29}$ In this phase II clinical trial, cetuximab also showed an ORR of $28 \%$ and a median progression-free survival (PFS) of 4.1 months with less toxicity in patients with lacSCC and mcSCC. ${ }^{29}$ Smaller prospective studies and patient cases have shown that higher ORR could be achieved when cetuximab is combined with chemotherapy and/or radiotherapy. In most cases, however, median PFS still remained short (Table 5). ${ }^{43,45,46,50}$ Cetuximab is recommended in Europe and Switzerland as a second-line treatment after first-line cemiplimab, combined with chemotherapy or radiotherapy. In a single-arm study of 16 patients with advanced cSCC, panitumumab, another anti-EGFR agent, showed similar efficacy (ORR of $31 \%$ with $19 \%$ partial response (PR), 12\% CR). ${ }^{51}$

\section{CHEMOTHERAPY AND ELECTROCHEMOTHERAPY}

No systemic chemotherapies have been approved in Switzerland to date for patients with advanced cSCC (level of evidence 3-4). ${ }^{7}$ In Europe, platinum agents (i.e. cisplatin or carboplatin), 5-fluorouracil, capecitabine, taxanes, bleomycin, methotrexate, adriamycin, doxorubicin, gemcitabine and ifosfamide have been used off-label either as monotherapy or polychemotherapy for advanced cSCC. ${ }^{7,43}$ Evidence to date suggests that polychemotherapies are more effective than monotherapies, ${ }^{7}$ with most responses being short-lived, followed by rapid recurrence, and failing to provide a curative effect. ${ }^{43}$ In a systematic review of $60 \mathrm{mcSCC}$ cases treated with cisplatin reported by Trodello et al. (2017), a CR was described in $22 \%$ of patient cases and PR in $23 \%$, resulting in an overall response of $45 \% .{ }^{43}$ Median disease-free survival for patients who attained CR was 14.6 months. ${ }^{43}$

ECT is a combination treatment used to reduce tumor progression in which a cytotoxic agent (usually bleomycin or cisplatin) is intravenously injected, followed by pulse application of an electric field into the cSCC tumor mass for enhanced drug delivery to cells. ${ }^{7,52}$ It has the advantage of high local tumor and bleeding control with minimal damage to normal tissue. ${ }^{753}$ In a European multicenter prospective study of the effectiveness of ECT in the treatment of skin cancer of the head and neck (EURECA), better responses with small lesions $(\leq 3 \mathrm{~cm})$, primary tumors, and naïve tumors $(\mathrm{p}<0.05$; level of evidence 3-4) were reported. ${ }^{33}$ At 2-months followup, CR was achieved in $55 \%$ of cSCC, PR in $24 \%$, SD in $15 \%$, and progression in $4 \% .{ }^{53}$ Bertino et al. (2016) concluded that ECT is an effective option for patients with head and neck cSCC when previous treatments had either failed or were not deemed suitable or declined by the patient. ${ }^{53}$ Overall, ECT was well tolerated and led to a significant improvement of quality of life for patients in this study. 7,53 
REFLECTION ON CURRENT EU TREATMENT GUIDELINES

Despite the approval of a new systemic treatment option for adult patients with metastatic or locally advanced cSCC who are not candidates for curative surgery or curative radiation in the $\mathrm{EU},{ }^{10}$ most $\mathrm{EU}$ national consensus management guidelines do not yet fully reflect recent evidence and the firstline change in systemic treatment for cSCC. In addition, it is not always clear when to use systemic therapies in lacSCC or mcSCC despite clear margins. Only the European Association of Dermato-Oncology (EADO), European Dermatology Forum (EDF) and European Organization for Research and Treatment of Cancer (EORTC) consensus guidelines (2020) provide a Grade A recommendation for first-line PD-1 inhibition with cemiplimab for patients with lacSCC or mcSCC who are not candidates for curative surgery or curative radiation. ${ }^{7}$ The 2019 German S3 guidelines for advanced cSCC patients were published prior to EMA approval of cemiplimab and therefore do not recommend any systemic treatment, except in the context of clinically controlled trials. ${ }^{54}$ The use of PD-1 inhibitors is mentioned as a novel therapeutic approach for inoperable cSCC, and initial data for cemiplimab is noted. ${ }^{9}$ Clinical trials underway with both cemiplimab and pembrolizumab are also mentioned in the German S3 guidelines. ${ }^{54}$ The National Comprehensive Cancer Network (NCCN Guidelines ${ }^{\circ}$ ) guidelines for the treatment of advanced cSCC were published a few months after the EMA approval of the first PD-1 inhibitor for the treatment of advanced cSCC. ${ }^{8}$ Systemic treatment for lacSCC and most cases of mcSCC is not recommended, but in an adapted footnote, immunotherapy (cemiplimab or clinical trial) should be considered if curative radiotherapy and curative surgery are not feasible. ${ }^{8}$

\section{CONCLUSIONS}

The recommendations herein are aimed to provide guidance to Swiss clinicians with the most up-to-date recommendations on how immunotherapy and other systemic therapies can be integrated into the treatment algorithm for advanced cSCC. In summary, an interdisciplinary approach is mandatory for patients with advanced cSCC to optimally manage their disease in the long-term. ${ }^{7-9}$ Treatment for primary low-risk cSCC remains surgical excision with post-operative margin assessment or Mohs micrographic surgery. Radiotherapy should be considered as curative treatment for inoperable primary common cSCC or for non-surgical candidates. Systemic therapy for advanced cSCC should be offered, whenever possible, as part of a clinical trial. In Switzerland, anti-PD-1 antibodies (i.e. cemiplimab) should now be the first-line systemic treatment for patients with mcSCC or lacSCC who are not candidates for curative surgery or radiation. Whilst there is clear agreement about the place of approved PD-1 inhibitors as the first-line systemic treatment, second-line treatment options are less clear. Cetuximab as well as chemotherapeutic agents may be discussed by the interdisciplinary tumor board as second-line treatment options for patients with advanced cSCC. Electrochemotherapy (ECT) may also be considered by the interdisciplinary tumor board and reserved for a very select number of patients in a few specialized centers. Best supportive care should be offered to patients with advanced disease to optimize symptom management and improve quality of life, and the frequency of follow-up visits and investigations for subsequent new cSCC should be determined by underlying risk characteristics.

The authors of these Swiss recommendations identified several outstanding questions that still need to be addressed in this field in the coming years: (1) how should PD-1 inhibitors be used in immunocompromised patients? (2) Can relative risks be reduced further with combination regimens? and (3) how effective is immunotherapy for advanced cSCC in the adjuvant and neoadjuvant setting ? ?-9,55 $^{7}$ Notably, ICIs are not recommended in the adjuvant/neoadjuvant setting outside of clinical trials since there is no data available; clinical trials are ongoing. In addition, several gaps have been identified in the existing EU guidelines for immunotherapy usage in cSCC, including limited use of checkpoint inhibitors in advanced cSCC patients taking immunosuppressive medications (e.g., organ transplant recipients, autoimmune disease).7-9,55 There is also no comprehensive information for systemic cSCC therapies in patients with underlying hematologic malignancies such as chronic lymphocytic leukemia (CLL). In early stage cSCC, there is currently no robust evidence to support the use of adjuvant or neoadjuvant systemic treatment for cSCC. It is, however, feasible that results from ongoing studies with the anti-PD-1 monoclonal antibodies, cemiplimab and pembrolizumab, may address some of these treatment gaps.

If patients decide against a therapy, our tasks include providing precise information about the tumor growth, expected and possible pain of the invasive bleeding tumor, as well as networking within a palliative service. 
The development and approval of the programmed-death protein 1 (PD-1) inhibitor cemiplimab has led to a new era in the systemic treatment of advanced cutaneous squamous cell carcinoma (cSCC). These Swiss recommendations provide guidance for the management of patients with lacSCC or mcSCC and will also help Swiss physicians in their decisionmaking.

- Systemic therapies should be considered for patients with advanced cSCC following an interdisciplinary tumor board discussion, and offered as part of a clinical trial whenever possible.

STATEMENTS ON CONFLICT OF INTERESTS

The authors declare that they hold advisory relationships with Sanofi that could be considered a potential conflict of interest related to the submitted manuscript.

\section{ACKNOWLEDGEMENT}

We thank Dr Ellen Heitlinger, $\mathrm{H}+\mathrm{O}$ communications Ltd., Zurich, Switzerland for her medical writing support of the manuscript (including writing, language editing, referencing, formatting, and proofreading), which was financially supported by Sanofi-Aventis (Switzerland) AG. Sanofi-Aventis did not have any decision-making role in the development of the manuscript and did not influence its content in any way.
- In Switzerland, the anti-PD-1 monoclonal antibody cemiplimab is recommended as the first-line systemic treatment for patients with distant metastatic or locally advanced cSCC who are not candidates for curative surgery or radiation.

- Ongoing clinical studies with immune checkpoint inhibitors will help to answer several critical questions involving the usage of anti-PD-1 antibodies in locally advanced and distant metastatic cSCC patients. 


\section{REFERENCES}

1. Leigh IM. Advanced cutaneous squamous cell carcinoma - a pressing case for treatment. J Eur Acad Dermatol Venereol. 2019;33 (Suppl_8):3-5.

2. Benzaquen M. Update on the anti-programmed cell death-1 receptor antibodies in advanced cutaneous squamouscell carcinoma. Dermatol Ther. 2020; 33(3): e13325.

3. Fitzgerald K, Tsay KK. Systemic therapy for advanced cutaneous squamous cell carcinoma. Semin Cutan Med cutaneous squamous cell carci

Surg. $2019 ; 38(1): E 67-74$.
4. Lomas A et al. A systematic review of worldwide inci4. Lomas A et al. A systematic review of worldwide inci-
dence of nonmelanoma skin cancer. Br J Dermatol. 2012; dence of nonmelan

5. Krebsliga. Patientenbroschüre Heller Hautkrebs. Krebsliga 2018. Available from: https://shop.krebsliga.ch/files/kls/webshop/PDFs/deutsch/heller-hautkrebs-011084012111.pdf.

6. Lieberherr $S$ et al. Evaluation of the National Skin Cancer Campaign: a Swiss experience of Euromelanoma. Swiss Med Wkly. 2017; 147: w14511.

7. Stratigos AJ et al. European interdisciplinary guideline on invasive squamous cell carcinoma of the skin: Part 2. Treatment. Eur J Cancer. 2020; 128: 83-102.

8. NCCN. NCCN Clinical Practice Guidelines in Oncology: Squamous Cell Skin Cancer. NCCN 2019. Available from: https://www.nccn.org/professionals/physician_gls/ default.aspx\#nmsc.

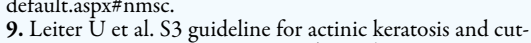
9. Leiter $\mathrm{U}$ et al. $\mathrm{S} 3$ guideline for actinic keratosis and cut-
aneous squamous cell carcinoma ( $\mathrm{CSCC}$ ) - short version, aneous squamous cell carcinoma ( $\mathrm{CSCC}$ ) - short version,
part 2: epidemiology, surgical and systemic treatment of part 2: epidemiology, surgical and systemic treatment of cSCC, follow-up, prevention and occupati
Dtsch Dermatol Ges. 2020; 18(4): 400-13.

10. LIBTAYO $^{\circ}$ (cemiplimab). Summary of product characteristics. Agency EM. Available from: https://www.ema. europa.eu/en/documents/product-information/libtayoepar-product-information en pdf

11. LIBTAYO ${ }^{\circ}$ (cemiplimab). Prescribing Information. FDA 2020. Available from: https://www.accessdata.fda. gov/drugsatfda_docs/label/2018/761097s000lbl.pdf.

12. Grob J-J et al. Pembrolizumab Monotherapy for Recur12. Grob -J et al. Pembrolizumab Monotherapy for RecurA Single-Arm Phase II Trial (KEYNOTE-629). J Clin Oncol. 2020; 38(25): 2916-25.

13. Howard J et al. The Oxford Levels of Evidence 2. 2011. Available from: https://www.cebm.net/index. aspx $?=5653$

14. Stratigos AJ et al. European interdisciplinary guideline on invasive squamous cell carcinoma of the skin: Part 1. epidemiology, diagnostics and prevention. Eur J Cancer. 2020; 128: 60-82.

15. García-Pedrero JM et al. Tumor programmed cell death ligand 1 expression correlates with nodal metastasis in pa-
tients with cutaneous squamous cell carcinoma of the head and neck. J Am Acad Dermatol. 2017; 77(3): 527-33.

16. García-Díez I et al. PD-L1 Expression is Increased in 16. Garcia-Diez I et al. PD-List Expression is Increased in Metastasizing Squamous Cell Carcinomas and Their
stases. Am J Dermatopathol. 2018; 40(9): 647-54. stases. Am J Dermatopathol. 2018; $40(9): 647-54$.
17. Kumar V et al. The Safety and Efficacy of Checkpoint 17. Kumar V et al. The Safety and Efficacy of Checkpoint
Inhibitors in Transplant Recipients: A Case Series and Inhibitors in Transplant Recipients: A Case Series and
Systematic Review of Literature. Oncologist. 2020; 25(6): Systematic

18. Gassmann D et al. Liver Allograft Failure After Nivolu-
mab Treatment-A Case Report With Systematic Literature Research. Transplant Direct. 2018; 4(8): e376.

19. De Bruyn P et al. Immune checkpoint blockade for organ transplant patients with advanced cancer: how far can we go? Curr Opin Oncol. 2019; 31(2): 54-64.

20. Fisher $J$ et al. Immune checkpoint inhibitor therapy in solid organ transplant recipients: A patient-centered systematic review. J Am Acad Dermatol. 2020; 82(6): 1490-500.

21. Sahu N, Grandis JR. New advances in molecular approaches to head and neck squamous cell carcinoma. Anticancer Drugs. 2011; 22(7): 656-64.

22. Hynes NE, Lane HA. ERBB receptors and cancer: the
cancer Drugs. complexity of targeted inhibitors. Nat Rev Cancer. 2005; 5(5): 341-54.

23. Maubec E et al. Immunohistochemical analysis of EGFR and HER-2 in patients with metastatic squamous cell carcinoma of the skin. Anticancer Res. 2005; 25(2B): 1205-10.

24. Cañueto J et al. Epidermal growth factor receptor expression is associated with poor outcome in cutaneous squamous cell carcinoma. Br J Dermatol. 2017; 176(5) squamous

25. Fogarty GB et al. Characterization of the expression and activation of the epidermal growth factor receptor in squamous cell carcinoma of the skin. Br J Dermatol. 2007; 156(1): $92-8$

26. Ridd K, Bastian BC. Somatic mutation of epidermal growth factor receptor in a small subset of cutaneous squamous cell carcinoma. J Invest Dermatol. 2010; 130(3) 901-3.

27. Forbes SA et al. COSMIC: exploring the world's knowledge of somatic mutations in human cancer. Nucleic Acid Res. 2015; 43(Database issue): D805-811.

28. Herbst RS, Langer CJ. Epidermal growth factor receptors as a target for cancer treatment: the emerging role of IMC-C 225 in the treatment of lung and head and neck cancers. Semin Oncol.2002:29(1 Suppl 4):27-36.

29. Maubec E et al. Phase II study of cetuximab as first-line 29. Maubec $\mathrm{E}$ et al. Phase II study of cetuximab as first-line single-drug therapy in patients with unresectable squamous
cell carcinoma of the skin. J Clin Oncol. 2011; 29(25): cell carci

3419-26.
30. Marti A et al. Complete Remission of Squamous Cell Carcinoma After Treatment With Panitumumab in a Patient With Cetuximab-Induced Anaphylaxis. JAMA Dermatol. 2016; 152(3): 343-5.

31. Alsaab HO et al. PD-1 and PD-L1 Checkpoint Signaling Inhibition for Cancer Immunotherapy: Mechanism, Combinations, and Clinical Outcome. Front Pharmacol. 2017; 8: 561 .

32. Migden MR et al. PD-1 Blockade with Cemiplimab in Advanced Cutaneous Squamous-Cell Carcinoma. N Engl I Med. 2018; 379(4): 341-51.

33. Migden MR et al. Cemiplimab in locally advanced cutaneous squamous cell carcinoma: results from an open-label, phase 2, single-arm trial. Lancet Oncol. 2020; 21(2): bel, phase

294-305.
34. Rischin D et al. Phase II study of cemiplimab in patients (pts) with advanced cutaneous squamous cell cacinoma (CSCC): Longer follow-up. JCO. 2020; 38(15 sSuppl_15): 10018-10018.

35. Migden $M$ et al. Health-Related Quality of Life (HRQL) in Patients with Advanced Cutaneous Squamous Cell Carcinoma (CSCC) Treated with Cemiplimab: Post Hoc Exploratory Analysis of a Phase 2 Clinical Trial. J of Skin. 2020; $4(6)$ : s122.

36. Maubec E et al. Phase II Study of Pembrolizumab As First-Line, Single-Drug Therapy for Patients With UnresecFirst-Line, Single-Drug Therapy for Patients With Unresec2020; 38(26): 3051-61.

37. Study of Pembrolizumab (MK-3475) in Adults With Recurrent/Metastatic Cutaneous Squamous Cell Carcinoma (cSCC) or Locally Advanced Unresectable cSCC (MK-3475-629/KEYNOTE-629). ClinicalTrials 2017 Available from: https://clinicaltrials.gov/ct2/show/ NCT03284424.

38. Study of Adjuvant Cemiplimab Versus Placebo After Surgery and Radiation Therapy in Patients With Hig Risk Cutaneous Squamous Cell Carcinoma. ClinicalTrials 2019. Available from: https://clinicaltrials.gov/ct2/show/ NCT03969004.

39. Pembrolizumab Versus Placebo Following Surgery and Radiation in Participants With Locally Advanced Cutaneous Squamous Cell Carcinoma (MK-3475-630/KEY NOTE-630). ClinicalTrials 2019. Available from: https:// NOTE-630). ClinicalTrials 2019. Available
clinicaltrials.gov/ct2/show/NCT03833167.

40. The Addition of Pembrolizumab to Postoperative
Radiotherapy in Cutaneous Squamous Cell Cancer of the Head and Neck. ClinicalTrials 2017. Available from https://clinicaltrials.gov/ct2/show/NCT03057613.

41. ERBITUX (cetuximab). Summary of product characteristics. Agency EM. Available from: https://www.ema. europa.eu/en/documents/product-information/erbituxepar-product-information_en.pdf.

42. Picard A et al. Association of Oncogenic Mutations in 42. Picard A et al. Association of Oncogenic Mutations in
Patients With Advanced Cutaneous Squamous Cell CarciPatients With Advanced Cutaneous Squamous Cell Carci-
nomas Treated With Cetuximab. JAMA Dermatol. 2017; nomas Treated
$153(4): 291-8$.

43. Trodello $C$ et al. Cisplatin and Cetuximab Treatment for Metastatic Cutaneous Squamous Cell Carcinoma: A Systematic Review. Dermatol Surg. 2017; 43(1): 40-9.

44. Conen KL et al. Cetuximab in metastatic squamous cell cancer of the skin: a Swiss case series. Dermatology. 2014 229(2): 97-101.

45. Dereure O et al. Efficacy and Tolerance of Cetuximab Alone or Combined with Chemotherapy in Locally Advanced or Metastatic Cutaneous Squamous Cell Carcinoma: An Open Study of 14 Patients. Dermatology. 2016; 232(6): 721-30.

46. Joseph $\mathrm{K}$ et al. Cetuximab-radiotherapy combination in the management of locally advanced cutaneous squamous cell carcinoma. J Med Imaging Radiat Oncol. 2019; 63(2): 257-63.

47. Giacchero D et al. Efficacy of cetuximab for unresectable or advanced cutaneous squamous cell carcinoma-report of eight cases. Clin Oncol (R Coll Radiol). 2011 23(10): 716-8

48. Samstein RM et al. Locally advanced and unresectable cutaneous squamous cell carcinoma: outcomes of concurrent cetuximab and radiotherapy. J Skin Cancer. 2014, 2014: 284582 .

49. Berliner JG et al. Response of Cutaneous Squamous Cell Carcinoma to Treatment With Cetuximab. Dermatol Surg. 2019; 45(2): 313-6.

50. Preneau $S$ et al. Efficacy of cetuximab in the treatment of squamous cell carcinoma. J Dermatolog Treat. 2014. 25(5): 424-7.

51. Foote MC et al. Phase II study of single-agent panitumumab in patients with incurable cutaneous squamous cell carcinoma. Ann Oncol. 2014; 25 (10): 2047-52

52. Gehl J et al. Updated standard operating procedures for electrochemotherapy of cutaneous tumours and skin metastases. Acta Oncol. 2018; 57(7): 874-82.

53. Bertino $G$ et al. European Research on Electrochemotherapy in Head and Neck Cancer (EURECA) project: Results of the treatment of skin cancer. Eur J Cancer. 2016, 63: 41-52.

54. Gutzmer R et al. Actinic Keratosis and Cutaneous Squamous Cell Carcinoma. Dtsch Arztebl Int. 2019; $116(37): 616-26$.

55. Patel R, Chang ALS. Immune Checkpoint Inhibitors for Treating Advanced Cutaneous Squamous Cell Carcinoma. Am J Clin Dermatol. 2019; 20(4): 477-82.

56. Seidl-Philipp M, Nguyen VA. Cutaneous squamous cel 56. Seidl-Philipp M, Nguyen VA. Cutaneous squamous cell carcinoma: Risk factors and new systemic treatment opt-
ons in advanced disease. memo. 2020; 13(1): 106-10. ons in advanced disease. memo. 2020; 13(1): 106-10.
57. Stratigos A et al. Diagnosis and treatment of invasive squamous cell carcinoma of the skin: European consensus-based interdisciplinary guideline. Eur J Cancer. 2015 51(14): 1989-2007.

58. Rischin D et al. Phase 2 study of cemiplimab in patients with advanced cutaneous squamous cell carcinoma (CSS) follow-up at 43 months. EADO; Virtual; 15th -17th April 2021. Oral Presentation. P-236.

59. Grob JJ et al. Phase 2 study of pembrolizumab monotherapy for locally advanced or recurrent/metastatic cutaneous squamous cell carcinoma. EADO; Virtual; 15th-17th April 2021. Oral Presentation. P-237. 